\title{
EDITORIAL
}

\section{Die ILMAC ist am Puls der Zeit}

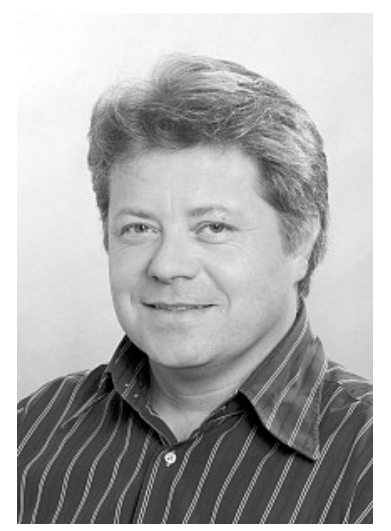

Das weniger Wichtige sei vorab gesagt: Mit gegen 600 Ausstellern (die endgültige Zahl ist bei Redaktionsschluss noch nicht bekannt) und einer prall gefüllten Halle 1 präsentiert sich die ILMAC 2007 grösser und repräsentativer als zuvor. - Folge einer überwiegend zufriedenen Kundschaft der letzten Durchführung, einer nachhaltig guten Konjunktur und dem einmaligen Vorteil, dass die Messe praktisch an Ihrem Arbeitsplatz stattfindet.

Wichtiger ist die hohe Aktualität der Themen, die an der ILMAC diskutiert werden: Das Scientific Forum der SCG ist mit dem Thema ,Energie und Rohstoffe' an Gravität und Aktualität kaum zu überbieten. Ein weiterer brandaktueller Themenschwerpunkt ist Paperless Lab Solutions mit zahlreichen Expertenvorträgen.

Pharmawasser wird mit täglichen Präsentationen und Round Tables in einem eigenen, unübersehbaren Pavillon in der Halle 1.0 thematisiert und last but not least zeigt die ILMAC das europaweit grösste Angebot für Reinraumtechnik mit ca. 40 Ausstellern und einem eigenen Themenpark des SRRT. - Damit übertreffen wir sogar aktuelle Spezialveranstaltungen, die sich diesem Thema exklusiv widmen.

Die hohe inhaltliche Aktualität macht den Puls der Zeit an der ILMAC fühlbar. Aber Achtung, die ILMAC dauert ,nur' vier Tage vom 25. - 28. September 2007. Lassen Sie sich die ILMAC nicht entgehen.

\section{Mit den besten Grüssen}

Christian Wenger

Leiter Industriefachmessen Messe Schweiz 